\title{
Advanced Adrenocortical Carcinoma: Current Perspectives on Medical Treatment
}

\author{
Authors \\ Alexandra Novais Araújoํㅜ, Maria João Bugalho, 2
}

\section{ABSTRACT}

Adrenocortical carcinoma (ACC) is a rare and aggressive malignancy. For stage I and II tumors, surgery is a curative option, but even in these cases recurrence is frequent. Practical guidelines advocate a combination of mitotane with etoposide, doxorubicin, and cisplatin as first-line therapy for metastatic adrenocortical carcinoma. However, this scheme presents limited efficacy and high toxicity. The use of Immune Checkpoint Inhibitors (ICI) and multi-Tyrosine Kinase Inhibitors (mTKI) has modified the approach of multiple malignancies. The expectation of their applicability on advanced adrenocortical carcinoma is high but the role of these new therapies persists unclear. This article provides a short summary of last years' findings targeting outcomes, limitations, and adverse effects of these new therapeutic approaches. The results of recent trials and case series pointed pembrolizumab as the most promising drug among these new therapies. It is the most often used $\mathrm{ICl}$ and the one presenting the best results with less related adverse effects when in comparison to the standard treatment with mitotane. Hereafter, the identification of specific molecular biomarkers or immune profiles associated with ICI or mTKI good response will facilitate the selection of candidates for these therapies. So far, microsatellite instability and Lynch Syndrome related germline mutations are suggested as predictive biomarkers of good response. Contrarywise, cortisol secretion has been associated with more aggressive ACC tumors and potentially poor responses to immunotherapy.

\section{Introduction}

Adrenocortical carcinoma (ACC) is a rare endocrine tumor with a poor prognosis. Recurrences are common even in patients with localized disease. The annual incidence is estimated to be between 0.7 and 2.0 cases per 1 million population. It can occur at any age, with a peak incidence between 40 and 50 years. ACC corresponds to $1.3 \%$ of all childhood cancer allowing to conclude for a higher relative incidence early in life. The incidence in children is particularly high in southern Brazil due to the high prevalence of a specific TP53 germline mutation [1]. In the adult as well as in the pediatric population, there is a predilection for the female gender [2-5].
ACC is, in most cases, a steroid hormone-producing tumor. The steroidogenic pattern is dominated by steroid precursor metabolites that are not measured by routine evaluation rather than end products of steroidogenesis [6]. Hypercortisolism (Cushing syndrome) or mixed Cushing and virilizing syndromes are the most common presentations in patients presenting with hormone excess; $30-40 \%$ of patients experience mass-effect symptoms, and $10 \%$ are incidentally found [3].

Stage at presentation is a crucial prognostic factor. Five-year survival is $60-80 \%$ for tumors limited to adrenal, $35-50 \%$ for locally advanced disease and much lower in case of metastatic disease ranging from $0 \%$ to $28 \%$. Nonetheless, this condition is very heter- 
ogeneous, and even patients with metastatic disease may have a long survival [7].

Surgical resection is the only curative option. However, it is only a realistic option for stage I and II tumors (rarely stage III). Even after apparently successful surgery, local or metastatic recurrence is frequent [3]. As a matter of fact, as many as $75-85 \%$ have a relapse after radical resection $[8,9]$. Recently, a nomogram incorporating age at diagnosis, $\mathrm{T}$ stage, $\mathrm{N}$ stage, and $\mathrm{M}$ stage has been proposed to predict overall survival of adult ACC patients after surgery [10].

Using the National Cancer Database (NCDB) and on Cox multivariate analysis, Tella SH et al. [11] concluded that increasing age, higher comorbidity index, high tumor grade, stage IV and no surgical therapy were associated with worse survival. Concerning the type of surgery - laparoscopic resection versus open adrenalectomy - in stage I-III disease, the presence of R0 resections was similar in either type of surgery.

Adjuvant treatment to reduce the risk of ACC recurrence after surgery is widely advocated, and several studies have taken place in an attempt to guide the optimal regimen [12]. Mitotane, [1-(2-chlorophenyl)-1-(4-chlorophenyl)-2,2-dichloroethane (o, $p^{\prime}$ DDD)], an analogue of the insecticide dichlorodiphenyltrichloroethane (DDT), has been used in the treatment of ACC since 1959 taking advantage of its cytotoxic effect on adrenal tissue and its potential to inhibit steroidogenesis [13]. Adjuvant mitotane may prolong recurrence-free survival in patients with radically resected adrenocortical carcinoma [14]. It is the only Food and Drug Administration (FDA) approved treatment of ACC. In advanced disease, mitotane is usually combined with etoposide, doxorubicin, and cisplatin (EDP) in a first-line attempt.

The response rates of mitotane monotherapy are estimated to be $10-30 \%$ [15]. A randomized phase III study of mitotane combined with EDP in patients with unresectable ACC without prior treatment (except mitotane) presented an ORR of $23.2 \%$, a disease-control rate (DCR) of $58.3 \%$, a median progressive free survival (mPFS) of 5 months and a median OS of 14.8 months [16].

The poor prognosis despite adjuvant therapy with mitotane with or without concurrent different chemotherapeutic regimens has led to the exploration of novel therapies [5, 17]. Abnormal activation of insulin-like growth factor receptor 1 (IGF1R) is one of the first and the most frequent molecular abnormalities described in sporadic adult ACC, with a very high prevalence. Therefore, the use of IGF1R inhibitors in association with mitotane appeared as an alternative treatment, however, with low therapeutic efficacy $[18,19]$.

The use of ICI has transformed the therapeutic approach of multiple human malignancies. Some of them, such as pembrolizumab, are already approved by FDA for all mismatch repair-deficient (MMRd) or high microsatellite instability (MSI-H) mutated solid tumors (independent of the histological findings) [20]. Some studies reported the use of mTKI in advanced ACC pointing for a modest efficacy [21,22]. Herein, we review new strategies for the management of patients with advanced ACC with a particular focus on immunotherapy: molecular rationale, outcomes, limitations, and adverse effects.

\section{Molecular markers and targeted therapies}

The majority of ACC are sporadic, but some cases occur as part of hereditary syndromes such as Li-Fraumeni Syndrome, BeckwithWiedemann Syndrome, Carney Complex or Multiple Endocrine Ne- oplasia type I [3]. Patients with Lynch Syndrome (LS) present a prevalence of ACC up to $3.2 \%$ [12].

As one of the rare cancer projects of The Cancer Genome Atlas (TCGA) a comprehensive genomic characterization of ACC was performed resulting in the identification of different genetic alterations from an expanded list of driver genes: IGF2, TP53, ZNFR3, CTNNB1, TERT, PRKAR1A, CCNE1, and TERF2[23]. The same study revealed frequent occurrence of massive DNA loss followed by whole-genome doubling (WGD) associated with ACC progression and reinforced the prognostic value of the methylation pattern.

A better understanding of the molecular pathogenesis of ACC offer hope that targeted therapies can be developed. The diversity of genomic alterations suggests the need for combined therapies.

\section{Tyrosine Kinase Inhibitors}

Molecular rationale

Selective and multi-kinase inhibitors are effective in the targeted treatment of various malignancies. They share the same mechanism of action however they differ from each other in the spectrum of targeted kinases. These drugs potentially inhibit a cascade of signaling events that regulate cell growth and angiogenesis. Overexpression of tyrosine kinase receptors was documented in ACC particularly vascular endothelial growth factors and receptors (VEGF/VEGFR), epidermal growth factor and receptor (EGF/EGFR) and insulin-like growth factor (IGF) system comprised by the IGF ligands (IGF-1, IGF-2, insulin) and their cell surface receptors (IGF$1 R$, IGF-2R and insulin receptor) [23-25].

Signaling pathways such as PI3K/AKT/mTOR and MAPK (Ras/ Raf/MEK/ERK) resulting in cell survival, proliferation and tumor growth, are activated through IGF-1R and EGF-R in ACC [25].

\section{Clinical trials}

Multikinase inhibitors like cabozantinib (C-Met, VEGFR2, AXL, and RET mTKI), levantinib (VEGFR1, VEGFR2, and VEGFR3 mTKI) and pazopanib (VEGFR, PDGFR and c-Kit) have been tested in advanced ACC with modest efficacy. Nonetheless, a small subset of patients, resistant to cytotoxic agents, may achieve partial remission [7, 22, 26].

A randomized phase III trial enrolling 139 patients showed no significant effect for linsitinib - an oral small molecule inhibitor of both insulin-like growth factor 1 receptor and the insulin receptor - compared to placebo in metastatic ACC [19]. The SIRAC-study (Sunitinib In Refractory Adrenocortical Carcinoma) showed that sunitinib had modest activity in advanced ACC [28].

Since mitotane is used in the vast majority of patients with advanced ACC and knowing that it has a strong and durable inducing effect on CYP3A4 activity likely to interfere with the efficacy of other therapies including TKI, analysis of TKI benefits has to take into account prior use of mitotane. Megerle et al. suggest the use of TKIs only when the levels of mitotane are $<2 \mathrm{mg} / \mathrm{l}$ [29].

\section{Immunotherapy}

Molecular rationale

Tumor mutational burden is characterized by the presence of more than 10 mutations per megabase. ACC has an intermediate tumor mutation burden particularly a high frequency of inactivating somatic or germline mutations of genes of the DNA mismatch repair (MMR) system that originate enhanced instability of microsatellite 
loci. Since the MMR-deficient cancers are associated with the presence of neoantigens, ACC was considered amenable to immunotherapy [30-33].

The immunotherapy is based on blocking key regulators of $\mathrm{T}$ cells (T-cell checkpoint molecules) using anti PD-1 or anti PD-L1. Programmed cell death-1 (PD-1) is an immune-checkpoint receptor expressed by $T$ cells, and programmed cell death ligand- 1 and 2 (PD-L1 and PD-L2) are expressed in the microenvironment of a number of cancers. An estimated $11 \%$ of ACCs express PD-L1 on tumor cell membranes, and $70 \%$ of tumor-infiltrating monocytes are PD-L1 positive [34]. The binding of PD-1 to PD-L1 or PD-L2 negatively regulates T-cell effector functions.

The over-expression of PD-L1 in several cancers as melanoma, non-small-cell lung carcinoma, and renal cell carcinoma have been regarded as good prognostic factor in terms of the response to PD-1 inhibitors therapy. However, there are studies reporting good overall response rates (ORR) to immunotherapy even in PD-L1 negative tumors. PD-L1 expression may not be the only determinant factor to immunotherapy response and more clinical trials are essential to determine the benefits of these drugs in advanced ACC [5, 35].

The hypercortisolism state of many ACC is pointed as a factor of immunotherapy resistance. The steroid phenotype is associated with lower overall survival (OS) when compared to non-functional ACCs [5]. Glucocorticoids have immunosuppressive function through inhibition of circulating and tumor-infiltrating immune cells. The presence of circulating and/or T lymphocytes is known to be correlated with favorable outcome on patients' OS in various malignancies as melanoma or lung cancer. These malignancies are highly responsive to immune checkpoint therapies [36]. In contrast, ACC displays the lowest pathological immune scores in cancer stromal cells infiltrates among different human neoplasms. But the real contribution of hypercortisolism state to the ineffectiveness of immunotherapy is uncertain [5].

Specific genetic alterations are possible indicators of immunotherapy resistance. $\beta$-Catenin gene (CTNNB1) and TP53 gene are frequently mutated in ACCs. Overactivation of $\beta$-catenin pathway and loss of p53 protein function are potential tumor-intrinsic factors that may alter the ability of ACC cells to recruit dendritic cells, leading to T-cell exclusion [2].

\section{Clinical trials}

In recent literature, we found 4 clinical trials using $\mathrm{ICI}$ to treat advanced ACC: a clinical trial phase Ib using avelumab - an anti-PD-L1 antibody; a phase II clinical trial using nivolumab - an anti-PD-1 antibody; and 2 phase II trials with pembrolizumab - another anti-PD-1 antibody [37-40] ( $\triangleright$ Table 1 ).

A multicenter, single-arm, open-label, phase 2 study to assess the ORR to nivolumab enrolled 10 patients with advanced ACC.

- Table 1 Clinical trials of immunotherapy in patients with advanced adrenocortical carcinoma.

\begin{tabular}{|c|c|c|c|c|}
\hline & Avelumab & Pembrolizumab & Nivolumab & Pembrolizumab \\
\hline ClinicalTrials. Gov Identifier & NCT01772004 & NCT02721732 & NCT02720484 & NCT02673333 \\
\hline Phase & $1 b$ & 2 & 2 & 2 \\
\hline $\mathrm{n}$ & 50 & 16 & 10 & 39 \\
\hline Female (\%) & $26(52 \%)$ & $8(50 \%)$ & 7 (70\%) & $23(59 \%)$ \\
\hline Age (median) & 50 & 48 & 57 & 62 \\
\hline Non-Functioning Tumor (\%) & NR & $6(37.5 \%)$ & $6(60 \%)$ & NR \\
\hline PDL 1/PD1 (\%) & $15(30 \%)$ & $0(0 \%)$ & $6(60 \%)$ & $7(18 \%)$ \\
\hline MSI/MMR-D (\%) & NR & $1(6.2 \%)$ & NR & $6(15 \%)$ \\
\hline$\geq 2$ lines of previous treatment (\%) & $37(74 \%)$ & $10(63 \%)$ & $3(30 \%)$ & NR \\
\hline Concomitant mitotane (\%) & $25(50 \%)$ & $0(0 \%)$ & $0(0 \%)$ & $0(0 \%)$ \\
\hline mPFS (months) & 2.6 & NR & 1.8 & 2.1 \\
\hline mOS (months) & 10.6 & NR & 21.2 & 24.9 \\
\hline Disease control rate & $48 \%$ & $57 \%$ & NA & $52 \%$ \\
\hline ORR & $6 \%$ & $14 \%$ & NA & $23 \%$ \\
\hline \multirow[t]{4}{*}{ Outcome } & PD 23 (46\%) & SD $7(43 \%)$ & PD 7 (70\%) & PD 15 (38\%) \\
\hline & SD 21 (42\%) & PD 5 (31\%) & SD 2 (20\%) & PR 9 (23\%) \\
\hline & PR 3 (6\%) & PR 2 (12.5\%) & PR $1(10 \%)^{*}$ & SD $7(18 \%)$ \\
\hline & CR $0(0 \%)$ & CR $0(0 \%)$ & CR $0(0 \%)$ & CR $0(0 \%)$ \\
\hline Final status (deaths) (\%) & $6(12 \%)$ & $10(63 \%)$ & $1(10 \%)$ & 20 (51\%) \\
\hline Follow-up (months) & 16.5 & 6.75 & 4.5 & 17.8 \\
\hline Reference & Le Tourneau et al. [40] & Habra et al. [38] & Carneiro et al. [39] & Raj et al. [37] \\
\hline
\end{tabular}


Nivolumab demonstrated a modest efficacy; just 1 patient achieved unconfirmed partial response (PR) [39].

Avelumab is a human IgG1 monoclonal antibody that specifically bind and block PD-L1. The JAVELIN trial assessed Its efficacy in patients with previously treated metastatic adrenocortical carcinoma with a DCR of $48 \%$ and ORR of $6 \%$. Results should be carefully interpreted because half of patients were treated with concomitant mitotane. From the 3 patients with PR, 2 were treated with double therapy [40].
The two trials with pembrolizumab presented better results than the trial with Avelumab. Pembrolizumab was used as single-agent therapy. The trials showed a promising efficacy regardless of tumor's hormonal function and MSI or PD-L1 status. A DCR of 57 and $52 \%$ and an ORR of 14 and $23 \%$ were described [ 37,38$]$. Isolated cases reporting the use of pembrolizumab reinforce the heterogeneity in terms of response [30] (

Combined therapies have also been assayed. The association of mitotane and pembrolizumab was used by Head et al. [41] in 6 pa-

- Table 2 Case series investigating immunotherapy or TKIs in patients with advanced adrenocortical carcinoma.

\begin{tabular}{|c|c|c|c|c|c|c|c|c|}
\hline Characteristics & Cabozantinib & $\begin{array}{l}\text { Pembroli- } \\
\text { zumab }\end{array}$ & $\begin{array}{l}\text { Pembroli- } \\
\text { zumab }\end{array}$ & $\begin{array}{l}\text { Pembroli- } \\
\text { zumab }\end{array}$ & $\begin{array}{l}\text { Pem- } \\
\text { broli- } \\
\text { zumab }\end{array}$ & $\begin{array}{l}\text { Nivolumab } \\
\text { plus } \\
\text { ipilimumab }\end{array}$ & $\begin{array}{l}\text { Lenvantinib } \\
\text { plus } \\
\text { pembroli- } \\
\text { zumab }\end{array}$ & $\begin{array}{l}\text { Cabozantinib } \\
\text { or levantinib } \\
\text { or pembroli- } \\
\text { zumab }\end{array}$ \\
\hline$n$ & 16 & 2 & 6 & 1 & 1 & 1 & 8 & 15 \\
\hline Female (\%) & $13(81 \%)$ & $2(100 \%)$ & $6(100 \%)$ & $1(100 \%)$ & $0(0 \%)$ & $0(0 \%)$ & $4(50 \%)$ & $7(47 \%)$ \\
\hline Age (median) & 42 & 34 & 44 & 58 & 29 & 38 & 38 & 43 \\
\hline Functioning Tumor (\%) & $13(81 \%)$ & NR & $3(50 \%)$ & $1(100 \%)$ & $1(100 \%)$ & $1(100 \%)$ & $3(37.5 \%)$ & $5(33 \%)$ \\
\hline Lynch syndrome & NR & NR & $2(33 \%)$ & $1(100 \%)$ & $0(0 \%)$ & $1(100 \%)$ & $0(0 \%)$ & $1(6.7 \%)$ \\
\hline PDL 1/PD1 & NR & $1(50 \%)$ & NR & $0(0 \%)$ & $1(100 \%)$ & NR & NR & NR \\
\hline MSI/MMR-D & NR & NR & $1(20 \%)$ & $1(100 \%)$ & $1(100 \%)$ & $1(100 \%)$ & NR & NR \\
\hline $\begin{array}{l}\geq 2 \text { lines of previous } \\
\text { treatment }\end{array}$ & At least 10 (62\%) & $2(100 \%)$ & $1(16 \%)$ & $0(0 \%)$ & $1(100 \%)$ & $0(0 \%)$ & $8(100 \%)$ & $14(93 \%)$ \\
\hline Concomitant mitotane & $0 \%$ & $0 \%$ & $6(100 \%)$ & $1(100 \%)$ & $1(100 \%)$ & $0(0 \%)$ & $0(0 \%)$ & $0(0 \%)$ \\
\hline \multirow[t]{2}{*}{ mPFS (months) } & 4 & NR & NR & NA & NA & NA & 5.5 & mTKI 6.3 \\
\hline & & & & & & & & Pem 1.4 \\
\hline \multirow[t]{2}{*}{ mOS (months) } & 14.5 & NR & NR & NA & NA & NA & NR & mTKI 17.2 \\
\hline & & & & & & & & Pem 5.3 \\
\hline \multirow[t]{2}{*}{ Disease control rate } & $50 \%$ & $50 \%$ & $100 \%$ & $0 \%$ & $100 \%$ & $100 \%$ & $25 \%$ & mTKI $63 \%$ \\
\hline & & & & & & & & Pem $17 \%$ \\
\hline \multirow[t]{2}{*}{ ORR } & $18.7 \%$ & $50 \%$ & $33 \%$ & $0 \%$ & $100 \%$ & $100 \%$ & $37.5 \%$ & mTKI $25 \%$ \\
\hline & & & & & & & & Pem $8 \%$ \\
\hline \multirow[t]{10}{*}{ Outcome } & PD 8 (50\%) & CR $1(50 \%)$ & SD $4(67 \%)$ & $\begin{array}{l}\text { PD } 1 \\
(100 \%)\end{array}$ & $\begin{array}{l}\text { PR } 1 \\
(100 \%)\end{array}$ & PR 1 (100\%) & PD 5 (62.5\%) & mTKI \\
\hline & SD 5 (31\%) & PD 1 (50\%) & PR 2 (33\%) & & & & PR 2 (25\%) & PD 3 (37,5\%) \\
\hline & PR 3 (18,7\%) & SD $0(0 \%)$ & PD $0(0 \%)$ & & & & SD $1(12.5 \%)$ & SD $3(37,5 \%)$ \\
\hline & CR 0 (0\%) & PR $0(0 \%)$ & CR $0(0 \%)$ & & & & CR 0 & PR 2 (25\%) \\
\hline & & & & & & & & CR $0(0 \%)$ \\
\hline & & & & & & & & Pem \\
\hline & & & & & & & & PD $10(83,4 \%)$ \\
\hline & & & & & & & & SD $1(8,3 \%)$ \\
\hline & & & & & & & & PR 1 (8,3\%) \\
\hline & & & & & & & & CR $0(0 \%)$ \\
\hline Final status (deaths) (\%) & $9(56 \%)$ & $1(50 \%)$ & $2(33 \%)$ & $1(100 \%)$ & $0(0 \%)$ & $0(0 \%)$ & $2(25 \%)$ & $10(67 \%)$ \\
\hline Follow-up (months) & NR & 26 & 21.3 & 4 & 6 & 24 & NR & NR \\
\hline Reference & $\begin{array}{l}\text { Kroiss } \\
\text { et al. [22] }\end{array}$ & $\begin{array}{l}\text { Mota } \\
\text { et al. [30] }\end{array}$ & $\begin{array}{l}\text { Head } \\
\text { et al. [41] }\end{array}$ & $\begin{array}{l}\text { Casey } \\
\text { et al. [46] }\end{array}$ & $\begin{array}{l}\text { Caccese } \\
\text { et al. [31] }\end{array}$ & $\begin{array}{l}\text { Nevgi } \\
\text { et al. [32] }\end{array}$ & $\begin{array}{l}\text { Bedrose } \\
\text { et al. [27] }\end{array}$ & $\begin{array}{l}\text { Miller } \\
\text { et al. [26] }\end{array}$ \\
\hline
\end{tabular}

MMR-D: Mismatch repair deficiency; MSI: Microsatellite instability; PDL1: Programmed death receptor 1 ligand; n: Number of patients; mPFS: Median progression-free survival; mOS: Median overall survival; ORR: Objective response rate; NR: Not reported; NA: Nnot applicable; PD: Progressive disease; SD: Stable disease; PR: Partial response; CR: Complete response; mTKI: Multi-Tyrosine Kinase Inhibitors; Pem: embrolizumab. 
- Table 3 Continued.

\begin{tabular}{|c|c|c|c|c|c|}
\hline \multirow[t]{2}{*}{ Adverse Event } & \multicolumn{2}{|c|}{ Raj et al. [37] } & \multicolumn{2}{|c|}{ Habra et al. [38] } & \multirow[t]{2}{*}{ Total n (\%) } \\
\hline & All Grades n (\%) & Grade $\geq 3 \mathrm{n}(\%)$ & All Grades n (\%) & Grade $\geq 3$ n (\%) & \\
\hline Increased AST/ALT & $9(23)$ & $4(10)$ & $2(13)$ & & $11(20)$ \\
\hline Fatigue & $8(20)$ & & $3(19)$ & & $11(20)$ \\
\hline Pruritus & $7(18)$ & & & & $7(12)$ \\
\hline Rash, maculo-papular & $3(8)$ & & $2(13)$ & & $5(9)$ \\
\hline Hypothyroidism & $3(8)$ & & $2(13)$ & & $5(9)$ \\
\hline Hypocalcemia & $4(10)$ & $1(3)$ & & & $4(7)$ \\
\hline Increased alkaline phosphatase & $4(10)$ & & & & $4(7)$ \\
\hline Nausea & $2(5)$ & & $1(6)$ & & $3(5)$ \\
\hline Anorexia & $1(3)$ & & $2(13)$ & & $3(5)$ \\
\hline Dry skin & $2(5)$ & & $1(6)$ & & $3(5)$ \\
\hline Lymphopenia & $2(5)$ & & & & $2(4)$ \\
\hline Increased creatinine & $2(5)$ & & & & $2(4)$ \\
\hline Hypoalbuminemia & $2(5)$ & $1(3)$ & & & $2(4)$ \\
\hline Hyperpigmentation & $2(5)$ & & & & $2(4)$ \\
\hline Chills & $2(5)$ & & & & $2(4)$ \\
\hline Oral mucositis & $1(3)$ & & $1(6)$ & & $2(4)$ \\
\hline Pneumonitis & $1(3)$ & & $1(6)$ & $1(6)$ & $2(4)$ \\
\hline Anemia & $1(3)$ & & $1(6)$ & & $2(4)$ \\
\hline Dyspnea & & & $1(6)$ & & $1(2)$ \\
\hline Arthralgia & & & $1(6)$ & & $1(2)$ \\
\hline Myalgia & & & $1(6)$ & & $1(2)$ \\
\hline Colitis & & & $1(6)$ & $1(6)$ & $1(2)$ \\
\hline Thrombocytopenia & $1(3)$ & & & & $1(2)$ \\
\hline Neutropenia & $1(3)$ & & & & $1(2)$ \\
\hline Hyperglycemia & $1(3)$ & & & & $1(2)$ \\
\hline Hypokalemia & $1(3)$ & $1(3)$ & & & $1(2)$ \\
\hline Hypophosphatemia & $1(3)$ & $1(3)$ & & & $1(2)$ \\
\hline Hypomagnesemia & $1(3)$ & $1(3)$ & & & $1(2)$ \\
\hline Hyponatremia & $1(3)$ & & & & $1(2)$ \\
\hline Increased bilirubin & $1(3)$ & & & & $1(2)$ \\
\hline Peripheral edema & $1(3)$ & & & & $1(2)$ \\
\hline Limbs edema & $1(3)$ & & & & $1(2)$ \\
\hline Pain & $1(3)$ & & & & $1(2)$ \\
\hline Decreased libido & $1(3)$ & & & & $1(2)$ \\
\hline Fever & $1(3)$ & & & & $1(2)$ \\
\hline Malaise & $1(3)$ & & & & $1(2)$ \\
\hline Other general toxicities & $1(3)$ & & & & $1(2)$ \\
\hline Hyperthyroidism & $1(3)$ & & & & $1(2)$ \\
\hline Adrenal insufficiency & $1(3)$ & & & & $1(2)$ \\
\hline Vomiting & $1(3)$ & & & & $1(2)$ \\
\hline Constipation & $1(3)$ & & & & $1(2)$ \\
\hline Diarrhea & $1(3)$ & & & & $1(2)$ \\
\hline Duodenitis & $1(3)$ & & & & $1(2)$ \\
\hline Alopecia & $1(3)$ & & & & $1(2)$ \\
\hline Skin and subcutaneous tissue disorder & $1(3)$ & & & & $1(2)$ \\
\hline Dry eyes & $1(3)$ & & & & $1(2)$ \\
\hline
\end{tabular}


- Table 3 Treatment related adverse events of Pembrolizumab trials.

\begin{tabular}{|c|c|c|c|c|c|}
\hline \multirow[t]{2}{*}{ Adverse Event } & \multicolumn{2}{|c|}{ Raj et al. [37] } & \multicolumn{2}{|c|}{ Habra et al. [38] } & \multirow[t]{2}{*}{ Total n (\%) } \\
\hline & All Grades n (\%) & Grade $\geq 3 \mathrm{n}$ (\%) & All Grades n (\%) & Grade $\geq 3 \mathrm{n}$ (\%) & \\
\hline Conjunctivitis & $1(3)$ & & & & $1(2)$ \\
\hline Dizziness & $1(3)$ & & & & $1(2)$ \\
\hline Infusion reaction & $1(3)$ & & & & $1(2)$ \\
\hline
\end{tabular}

tients and a partial response was observed in 2 of them. The association of pembrolizumab and levantinib conducted to a partial response in 1 out of 8 heavily pre-treated patients [27]. The rationale for this type of combination is that MKI modulate a variety of interferon-signaling related genes and in this way activate CD8 + T cells in tumoral microenvironment.

The association of pembrolizumab and chemotherapy (i. e., cyclophosphamide or gemcitabine/doxetaxel) was reported by Miller et al. [26] in 4 patients and all had disease progression.

A better characterization of the genomic, molecular and immune profiles of the good responders will facilitate the selection of candidates for these therapies. So far, it seems that microsatellite instability and Lynch Syndrome related germline mutations may be predictive biomarkers of response to immunotherapy. Germline mutations in the CDKN2A gene might also be associated with a favorable response to immunotherapy $[2,26,42]$. On the other hand, cortisol secretion has been associated with more aggressive ACC tumors and potentially poor responses to immunotherapy [43].

\section{Adverse Effects (AE)}

In general, the adverse effects observed in patients with advanced ACC treated with $\mathrm{TKI}$ or ICl are the same reported in the setting of other malignancies [39, 44, 45]. However, the frequent association with mitotane that has a toxicity profile including hepatic, gastrointestinal, neurological, and hematologic effects increases the risk of adverse effects. Patients receiving concomitant mitotane have a higher rate of grade $\geq 3$ treatment related adverse effects (TRAEs) than those with single therapy, particularly liver enzymes elevation [40].

From the literature, the occurrence of TRAEs in ACC patients is estimated to range between $58.9 \%$ and $100 \%$ but in most of the cases the severity of symptoms were mild without the need to stop treatment. A death, possible related to pembrolizumab, was reported [46].

The TRAEs observed in 55 patients with pembrolizumab, retrieved from the literature, are outlined in $>$ Table 3 . Grade 3 or higher were observed in 11 patients (20\%) which is much lower than the rate of $58 \%$ reported in patients submitted to EDP treatment [37]. Nonetheless, close clinical and biochemical monitoring are indicated.

\section{Conclusions}

ACC is a rare, aggressive and heterogeneous endocrine tumor. The standard treatment with mitotane with or without EDP has limited efficacy and important AEs. Recurrence is the most common outcome. Early recurrence is associated with an advanced stage of disease at diagnosis, incomplete surgical resection, cortisol production, and a few genetic alterations.

The low incidence of the disease and high cost of clinical trials are the major constraints in the search for improved treatment strategies. That is why new concepts for clinical trials such as registry-based randomized trials, as proposed by the European Network for the Study of Adrenal Tumours (ENSAT) [47], will probably facilitate future studies.

Pembrolizumab is the most often used checkpoint inhibitor and the one that showed the best results in patients with advanced ACC. In some series, the ORR reached $33 \%$ in patients in whom other previous treatments had failed. Identification of biomarkers allowing to select the candidates for immunotherapy are likely to improve results. Putative candidates might be those with mutations in DNA repair-related genes [30]. Whether the high intratumoral concentrations of glucocorticoids might interfere in the response to this class of medications remains to be answered.

Clinical trials exposed weaknesses of the single pathway, "onesize-fits-all" therapy, that could be anticipated, due to the high mutational burden of ACC, thus, reinforcing the need for combined therapies.

In summary, immunotherapy and/or multi-kinase inhibitors may be a salvage therapeutic option to ACC patients with limited options. However, this approach requires the improved patient selection and future studies aiming to identify biomarkers that predict response.

While awaiting an effective treatment for this orphan disease, a prompt diagnosis of relapse remains the best alternative to care for the patients. Therefore, development of liquid biopsies, including steroid metabolomics, cell-free DNA or microRNA, to use on a clinical ground are promising tools for the postoperative follow-up and early detection of relapse. It is conceivable that a biochemical detection of relapse, prior to the evidence of structural disease will have a positive impact on treatment efficacy. 


\section{Conflict of Interest}

The authors declare that they have no conflict of interest.

\section{References}

[1] Custódio G, Komechen H, Figueiredo FRO et al. Molecular epidemiology of adrenocortical tumors in southern Brazil. Mol Cell Endocrinol 2012; 351: 44-51

[2] Altieri B, Ronchi CL, Kroiss M et al. Next-generation therapies for adrenocortical carcinoma. Best Pract Res Clin Endocrinol Metab 2020; 34: 101434

[3] Fassnacht M, Dekkers OM, Else T et al. European Society of Endocrinology Clinical Practice Guidelines on the management of adrenocortical carcinoma in adults, in collaboration with the European Network for the Study of Adrenal Tumors. Eur J Endocrinol 2018; 179: G1-G46

[4] Else T, Kim AC, Sabolch A et al. Adrenocortical carcinoma. Endocr Rev 2014; 35: 282-326

[5] Fiorentini C, Grisanti S, Cosentini D et al. Molecular drivers of potential immunotherapy failure in adrenocortical carcinoma. J Oncol 2019; 2019: 1-7

[6] Chortis V, Bancos I, Nijman T et al. Urine steroid metabolomics as a novel tool for detection of recurrent adrenocortical carcinoma. J Clin Endocrinol Metab 2020; 105: e307-e318

[7] Brabo EP, Moraes AB, Neto LV. The role of immune checkpoint inhibitor therapy in advanced adrenocortical carcinoma revisited: Review of literature. J Endocrinol Invest 2020; 43: 1531-1542

[8] Pommier RF, Brennan MF. An eleven-year experience with adrenocortical carcinoma. Surgery 1992; 112: 96370. discussion 70-71

[9] Stojadinovic A, Ghossein RA, Hoos A et al. Adrenocortical carcinoma: Clinical, morphologic, and molecular characterization. J Clin Oncol 2002; 20: 941-950

[10] Kong J, Zheng J, Cai J et al. A nomogram for individualized estimation of survival among adult patients with adrenocortical carcinoma after surgery: a retrospective analysis and multicenter validation study. Cancer Commun 2019; 39: 80

[11] Tella SH, Kommalapati A, Yaturu S et al. Predictors of survival in adrenocortical carcinoma: An analysis from the National Cancer Database. J Clin Endocrinol Metab 2018; 103: 3566-3573

[12] Bedrose S, Daher M, Altameemi L et al. Adjuvant Therapy in Adrenocortical Carcinoma: Reflections and Future Directions. Cancers (Basel) 2020; 12: 508

[13] Bergenstal D, Lipsett MB, Moy R et al. Regression of Adrenal Cancer and Suppression of Adrenal Function in Man by o,p'-DDD11. Biological Activities of Steroids in Relation to Cancer. Pincus G, Vollmer EP. Maryland: Academic Press, 1960: 463-475

[14] Terzolo M, Angeli A, Fassnacht M et al. Adjuvant Mitotane Treatment for Adrenocortical Carcinoma. N Engl J Med 2007; 356: 2372-2380

[15] Kulk M, Shah M, Benson A et al. NCCN Clinical Practice Guidelines in Oncology:Neuroendocrine tumors. J Natl Compr Canc Netw 2015;13(1): 78-108

[16] Fassnacht M, Terzolo M, Allolio B et al. Combination Chemotherapy in Advanced Adrenocortical Carcinoma. N Engl J Med 2012; 366: 89-97

[17] Sperone P, Ferrero A, Daffara F et al. Gemcitabine plus metronomic 5 -fluorouracil or capecitabine as a second-/third-line chemotherapy in advanced adrenocortical carcinoma: a multicenter phase II study. Endocr Relat Cancer 2010; 17: 445-453

[18] Barlaskar FM, Spalding AC, Heaton JH et al. Preclinical targeting of the type I insulin-like growth factor receptor in adrenocortical carcinoma. J Clin Endocrinol Metab 2009; 94: 204-212
[19] Fassnacht M, Berruti A, Baudin E et al. Linsitinib (OSI-906) versus placebo for patients with locally advanced or metastatic adrenocortical carcinoma: A double-blind, randomised, phase 3 study. Lancet Oncol 2015; 16: 426-435

[20] Le DT, Durham JN, Smith KN et al. Mismatch repair deficiency predicts response of solid tumors to PD-1 blockade. Science 2017; 357: 409-413

[21] Zhuang J, Wang D, Wu R et al. Sunitinib monotherapy instead of mitotane combination therapy for the treatment of refractory adrenocortical carcinoma. Int J Urol 2015; 22: 1079-1081

[22] Kroiss M, Megerle F, Kurlbaum M et al. Objective response and prolonged disease control of advanced adrenocortical carcinoma with cabozantinib. J Clin Endocrinol Metab 2020; 105 dgz318

[23] Zheng S, Cherniack AD, Dewal N et al. Comprehensive pan-genomic characterization of adrenocortical carcinoma. Cancer Cell 2016; 29 : 723-736

[24] Mariniello B, Rosato A, Zuccolotto G et al. Combination of sorafenib and everolimus impacts therapeutically on adrenocortical tumor models. J Endocr Relat Cancer 2012; 19: 527

[25] Angelousi A, Kyriakopoulos G, Nasiri-Ansari N et al. The role of epithelial growth factors and insulin growth factors in the adrenal neoplasms. Ann Transl Med 2018; 6: 253

[26] Miller KC, Chintakuntlawar AV, Hilger C et al. Salvage therapy with multikinase inhibitors and immunotherapy in advanced adrenal cortical carcinoma. J Endocr Soc 2020; 4: bvaa069

[27] Bedrose S, Miller KC, Altameemi L et al. Combined lenvatinib and pembrolizumab as salvage therapy in advanced adrenal cortical carcinoma. J Immunother Cancer 2020; 8: e001009

[28] Kroiss M, Quinkler M, Johanssen $S$ et al. Sunitinib in refractory adrenocortical carcinoma: a phase II, single-arm, open-label trial. ] Clin Endocrinol Metab 2012; 97: 3495-3503

[29] Megerle F, Kroiss M, Hahner S et al. Advanced Adrenocortical Carcinoma - What to do when First-Line Therapy Fails? Exp Clin Endocrinol Diabetes 2019; 127: 109-116

[30] Mota JM, Sousa LG, Braghiroli MI et al. Pembrolizumab for metastatic adrenocortical carcinoma with high mutational burden: Two case reports. Medicine (Baltimore) 2018; 97: e13517

[31] Caccese M, Ceccato F, Fassan M et al. Letter to Editor: Reply to R.T. Casey (Semin Oncol 2018; 45: 151-155). Semin Oncol 2019; 46: 104-105

[32] Nevgi A, Klein O, Cheung AS. Sustained remission of Lynch syndrome-associated metastatic adrenocortical carcinoma following checkpoint inhibitor therapy-associated multiorgan autoimmunity. Clin Endocrinol 2020; 93: 214-216

[33] Gara SK, Lack J, Zhang L et al. Metastatic adrenocortical carcinoma displays higher mutation rate and tumor heterogeneity than primary tumors. Nat Commun 2018; 9: 4172

[34] Khoja L, Butler MO, Kang SP et al. Pembrolizumab. J Immunother Cancer 2015; 3: 36

[35] Naing A, Meric-Bernstam F, Stephen B et al. Phase 2 study of pembrolizumab in patients with advanced rare cancers. J Immunother Cancer 2020; 8: e000347

[36] Landwehr L-S, Altieri B, Schreiner J et al. Interplay between glucocorticoids and tumor-infiltrating lymphocytes on the prognosis of adrenocortical carcinoma. J Immunother Cancer 2020; 8: e000469

[37] Raj N, Zheng Y, Kelly V et al. PD-1 Blockade in Advanced Adrenocortical Carcinoma. J Clin Oncol 2020; 38: 71-80

[38] Habra MA, Stephen B, Campbell M et al. Phase II clinical trial of pembrolizumab efficacy and safety in advanced adrenocortical carcinoma. J Immunother Cancer 2019; 7: 253 
[39] Carneiro BA, Konda B, Costa RB et al. Nivolumab in Metastatic Adrenocortical Carcinoma: Results of a Phase 2 Trial. J Clin Endocrinol Metab 2019; 104: 6193-6200

[40] Le Tourneau C, Hoimes C, Zarwan C et al. Avelumab in patients with previously treated metastatic adrenocortical carcinoma: phase $1 \mathrm{~b}$ results from the JAVELIN solid tumor trial. J Immunother Cancer 2018; 6: 111

[41] Head L, Kiseljak-Vassiliades K, Clark T] et al. Response to Immunotherapy in Combination With Mitotane in Patients With Metastatic Adrenocortical Cancer. J Endocr Soc 2019; 3: 2295-2304

[42] Andrews MC, Wargo JA. Immunotherapy resistance: the answers lie ahead - not in front - of us. J Immunother Cancer 2017; 5: 10

[43] Puglisi S, Perotti P, Pia A et al Adrenocortical Carcinoma with Hypercortisolism. Endocrinol Metab Clin North Am 2018; 47: 395-407
[44] Kwok G, Yau TCC, Chiu JW et al. Pembrolizumab (Keytruda). Hum Vaccin Immunother 2016; 12: 2777-2789

[45] Markowitz JN, Fancher KM. Cabozantinib: A Multitargeted Oral Tyrosine Kinase Inhibitor. Pharmacotherapy 2018; 38: 357-369

[46] Casey RT, Giger O, Seetho I et al. Rapid disease progression in a patient with mismatch repair-deficient and cortisol secreting adrenocortical carcinoma treated with pembrolizumab. Semin Oncol 2018; 45: $151-155$

[47] Crona J, Baudin E, Terzolo M et al. ENSAT Registry-Based Randomized Clinical Trials for Adrenocortical Carcinoma. Eur J Endocrinol 2021; 184: R51-R59 